\title{
Effect of a local nonlinearity on the light dynamics around an exceptional point: A quantitative analysis
}

\author{
Jyotismita Doley $\odot,{ }^{1}$ Arnab Laha $\odot,{ }^{1,2}$ Sibnath Dey $\odot,{ }^{1}$ and Somnath Ghosh $\oplus^{1, *}$ \\ ${ }^{1}$ Department of Physics, Indian Institute of Technology Jodhpur, Rajasthan 342037, India \\ ${ }^{2}$ Institute of Radiophysics and Electronics, University of Calcutta, Kolkata 700009, India
}

(Received 29 February 2020; accepted 29 May 2020; published 22 June 2020)

\begin{abstract}
Time-asymmetric state evolution due to the dynamical encirclement around a second-order exceptional point has garnered enormous attention for the topological study of various photonic structures. The selective modeconversion in an optical waveguide can help in the designing of various integrated photonics devices. Here we explore a dual-mode planar optical waveguide and study the beam dynamics with the onset and offset of Kerr nonlinearity together with the encirclement of the identified exceptional point in the parameter space. The onset of the same amount of focusing and defocusing type nonlinearity in the waveguide gives two different outputs for a unidirectional propagation of light, beyond the chiral aspect of the device.
\end{abstract}

DOI: 10.1103/PhysRevResearch.2.023371

\section{INTRODUCTION}

Non-Hermitian quantum mechanics, which can more accurately describe an open system, has garnered enormous attention in the field of quantum-inspired photonic systems. Such open systems have abundant physical aspects due to their interaction with their surroundings. Non-Hermitian quantum mechanics provides a formalism to define any open system by an effective Hamiltonian [1]. The appearance of hidden singularities called the exceptional points (EPs) is one of the intriguing topological features of such systems [2,3]. A second-order EP is a branch point singularity in parameter space where two eigenvalues and their corresponding eigenvectors of a system's Hamiltonian coalesce simultaneously, thereby creating a defect [4-6]. Such anomalous behavior of $\mathrm{EP}$ has given rise to various phenomena such as flip of states [7-14], asymmetric mode conversion [15-18], topological energy transfer [19], lasing and antilasing [20], unidirectional light reflection and transmission [21], EP-aided enhanced sensing [22], nonreciprocity [23-25], stopping of light [26], and cross-polarization mode coupling [19,27-29].

A stroboscopic encirclement around an EP in parameter space allows adiabatic state exchange between a pair of coupled eigenmodes [7-9,12-14,17,30]. However, a dynamical parametric encirclement around an EP results in the breakdown of the adiabaticity during state evolutions where the two coupled modes evolve with different decay rates [31,32]. At the end of the encirclement, the state with an average lower decay rate dominates. Clockwise and anticlockwise

\footnotetext{
*somiit@ rediffmail.com

Published by the American Physical Society under the terms of the Creative Commons Attribution 4.0 International license. Further distribution of this work must maintain attribution to the author(s) and the published article's title, journal citation, and DOI.
}

parametric encirclements yield different dominating output modes irrespective of the choice of input modes $[15,17,33]$. Apart from the already reported dual-mode systems [15-17,34] with asymmetric mode conversion, the realization of different dominating modes at the same output port due to a single encirclement direction is more practical for integrated photonic devices from designing aspects and is yet to be explored. Apart from the already-reported dual-mode systems with chirality driven asymmetric mode conversion, a more practical scenario owing to an integrated device application is the exhibition of the nonchiral asymmetric mode conversion for unidirectional propagation of light in the same device. Such nonchiral asymmetric mode conversion can be realized with the onset of nonlinearities in the system. Nonlinearities in systems hosting an EP exhibit intriguing phenomena such as nonreciprocal optical transmission [23,24], enhanced second-harmonic generation [35], and so on.

In this paper, to address the proposition mentioned above, we report a gain-loss assisted dual-mode optical waveguide hosting a second-order EP in the parameter plane. Considering a length-dependent gain-loss variation along the propagation direction of the waveguide, the embedded EP has been dynamically encircled to achieve a chirality driven asymmetric mode conversion scheme, where, depending on the direction of light propagation, light is converted to a specific dominating output mode, irrespective of the choice of input modes. Now introducing the local Kerr-type nonlinearity as an additional parameter, we show that beyond the conventional chiral aspect of the device in the vicinity of an EP, the interplay of EP and nonlinearity can additionally possess a special unidirectional asymmetric mode conversion scheme. Here, in the presence of a certain amount of nonlinearity above a particular threshold, light is converted to a specific dominating mode while propagating in a particular direction, where the same amount of focusing and defocusing nonlinearities results in different dominating outputs in the same direction, respectively. The proposed scheme should be suitable to design 
asymmetric mode-converters without using the chiral aspect of the device.

\section{ANALYTICAL MODEL ANALOGOUS TO A DUAL-MODE PLANAR WAVEGUIDE}

The appearance of an EP in a dual-mode planar waveguide can analytically be realized by considering a two-level nonHermitian Hamiltonian as

$$
\mathcal{H}=\left(\begin{array}{cc}
\beta_{p}+i \alpha_{p} & \eta \\
\eta^{*} & \beta_{q}+i \alpha_{q}
\end{array}\right),
$$

where, $\beta_{p}$ and $\beta_{q}$ are the real propagation constants with the respective decay rates $\alpha_{p}$ and $\alpha_{q}$. For simplification, we define two new terms $\widetilde{\beta}_{j}=\beta_{j}+i \alpha_{j}(j=p, q)$ as complex propagation constants. The off-diagonal terms $\eta$ and its complex conjugate $\eta^{*}$ appear as perturbation to modulate the coupling between $\widetilde{\beta}_{p}$ and $\widetilde{\beta}_{q}$. Now, the eigenvalues of the Hamiltonian $\mathcal{H}$ can be written as

$$
\beta_{ \pm}=\frac{\widetilde{\beta}_{p}+\widetilde{\beta}_{q}}{2} \pm \sqrt{\left(\frac{\widetilde{\beta}_{p}-\widetilde{\beta}_{q}}{2}\right)^{2}+|\eta|^{2}} .
$$

In Eq. (2), we bring up two individual replacements e.g. $\widetilde{\beta}_{\text {av }}=$ $\left(\widetilde{\beta}_{p}+\widetilde{\beta}_{q}\right) / 2$ and $\delta \widetilde{\beta}=\left(\widetilde{\beta}_{p}-\widetilde{\beta}_{q}\right) / 2$, which gives a revised form of $\beta_{ \pm}$as

$$
\beta_{ \pm}=\widetilde{\beta}_{\mathrm{av}} \pm \sqrt{(\delta \widetilde{\beta})^{2}+|\eta|^{2}} .
$$

Here, two coupled eigenvalues coalesce with $\beta_{+}=\beta_{-}$, which gives the condition for occurrence of an EP. Thus, in the complex $\eta$-plane, an EP appears at the critical point $\eta_{\mathrm{c}}=$ $\pm i \delta \widetilde{\beta}_{\mathrm{c}}$, whereas the position of the identified EP can be accessed by real and imaginary parts of $\delta \widetilde{\beta}$ (where, $\delta \widetilde{\beta} \equiv$ $\delta \beta+i \delta \alpha)$ with the fulfillment of the conditions $\beta_{p}=\beta_{q}$ and $\eta=\left|\alpha_{p}-\alpha_{q}\right| / 2$.

While implementing the above analytical scheme in a physical dual-mode waveguide system, the coupling terms ( $\eta$ and $\eta^{*}$ ) can be introduced in terms of optical gain-loss. Now, if we consider the intensity-dependent local nonlinearity in the waveguide, the parameters $\beta_{j}$ and $\alpha_{j}(j=p, q)$ in the corresponding Hamiltonian $\mathcal{H}$ varies as a function of signal intensity $(I)$, i.e., $\beta_{j}=\beta_{j}(I)$ and $\alpha_{j}=\alpha_{j}(I)$. Typically, we can define a threshold intensity $I_{\mathrm{th}}$, where only for $I>I_{\mathrm{th}}$, the effect of nonlinearity comes into the picture, and then simultaneously with the introduced gain-loss, the nonlinearity factor indeed affects the overall interaction phenomenon. There would be no effect of nonlinearity for $I<I_{\mathrm{th}}$. Such interplay between gain-loss and nonlinearity will be discussed later in more context.

\section{OPTICAL WAVEGUIDE HOSTING AN ASYMMETRIC MODE CONVERSION SCHEME}

We design a planar optical waveguide, as shown schematically in Fig. 1(a). We consider the $z$-axis as the propagation direction for the light beam and $x$-axis as the transverse direction. The region $-W / 2<x<W / 2$ of the waveguide consists of a core of passive refractive index $n_{h}=1.5$, surrounded by cladding with passive refractive index $n_{l}=1.46$, respectively. By normalizing $\omega=1$, we set the width of the

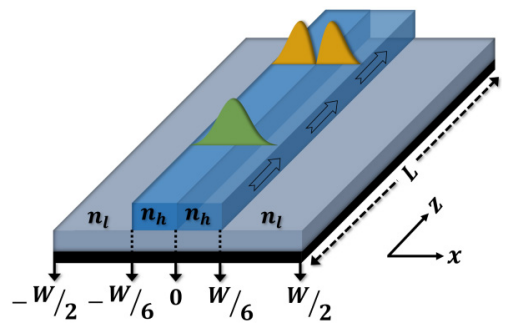

(a)

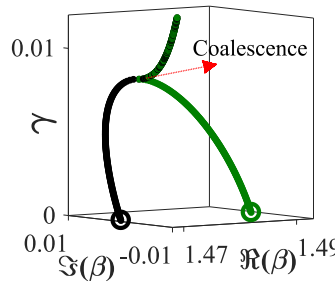

(c)

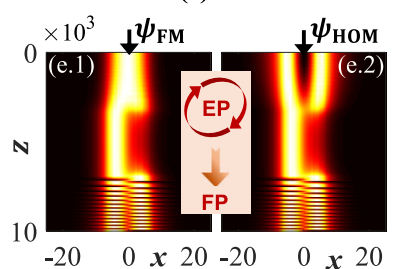

(e)

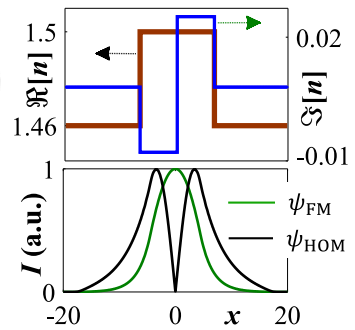

(b)

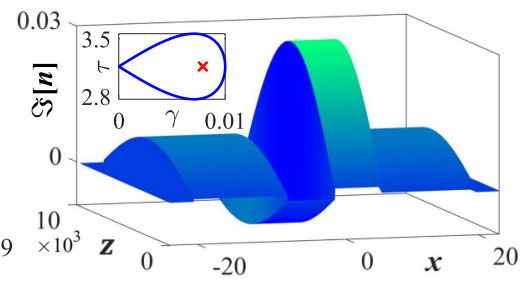

(d)

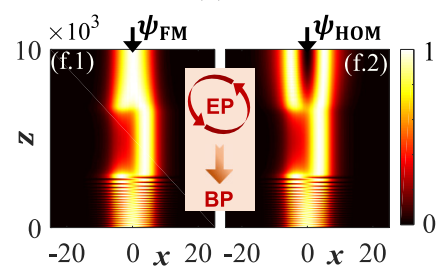

(f)

FIG. 1. Waveguide hosting a dynamical EP-encirclement scheme. (a) Schematic of the designed optical waveguide with transverse $x$-axis. Propagation is considered along the $z$-axis. (b) Transverse refractive index profile $n(x)$ showing $\operatorname{Re}(n)$ (solid brown line) and $\operatorname{Im}(n)$ (upper panel) at specific $\gamma=0.008$ and $\tau=3.179$. Normalized output field intensity profiles of the supported modes $\left(\psi_{\mathrm{FM}}\right.$ and $\left.\psi_{\mathrm{HOM}}\right)$. (c) Trajectories of complex $\beta$ values with respect to $\gamma$ for a specific value of $\tau=3.179$, where the two $\beta$ s coalesce near $\gamma=0.008$. (d) Length-dependent variation of $\operatorname{Im}(n)$ after mapping the chosen parameter as shown in the inset. Chosen topological structure of the waveguide with simultaneous variation of $\gamma$ and $\tau$ around the identified EP (inset). (e) Beam evolution for the clockwise dynamical encirclement scheme. (e.1) Evolution of $\psi_{\mathrm{FM}}$ to $\psi_{\mathrm{HOM}}$. (e.2) Evolution of $\psi_{\mathrm{HOM}}$ to $\psi_{\mathrm{HOM}}$. (f) Beam evolution for the anticlockwise dynamical encirclement scheme. (f.1) Evolution of $\psi_{\mathrm{FM}}$ to $\psi_{\mathrm{FM}}$. (f.2) Evolution of $\psi_{\mathrm{HOM}}$ to $\psi_{\mathrm{FM}}$.

structure $W=40 \lambda / 2 \pi=40$ and the length $L=10 \times 10^{3}$ in a dimensionless unit. For the chosen set of characteristic parameters, the waveguide supports only the fundamental mode (FM) and the first higher-order mode (HOM). Now the non-Hermitian characteristic is introduced in the system by a specific transverse distribution of an unbalanced gain-loss profile, where the complex profile of $n(x)$ for a specific crosssection of the waveguide can be written as follows:

$$
n(x)= \begin{cases}n_{l}+i \gamma & \text { for } W / 6 \leqslant|x| \leqslant W / 2, \\ n_{h}-i \gamma & \text { for }-W / 6 \leqslant x \leqslant 0 \\ n_{h}+i \tau \gamma & \text { for } 0 \leqslant x \leqslant W / 6\end{cases}
$$

Here, $\gamma$ and $\tau$ are the two control parameters that represent the gain-coefficient and the loss-to-gain ratio, respectively. The overall refractive index profile for a specific cross-section 
of the waveguide as represented by Eq. (4) is shown in the upper panel of Fig. 1(b). We can modulate non-Hermiticity in the designed waveguide by tuning these parameters independently according to the Kramers-Kronig causality relation at a single operating frequency [36]. A prototype of the proposed planar waveguide structure can be fabricated by thin film deposition of glass material $\left(n_{h}=1.50\right)$ over a thick silicaglass substrate $\left(n_{l}=1.46\right)$. The patterned gain-loss profile can be achieved by a standard photolithography technique or by controlled doping of gain or lossy materials with a state-of-art-fabrication technique.

In the proposed waveguide, the values of the propagation constants $(\beta)$ of the quasiguided modes are calculated by solving the scalar modal equation $\left[\partial_{x}^{2}+n^{2}(x) \omega^{2}-\beta^{2}\right] \psi(x)=0$, which corresponds to a steady-state mode profile $\psi(x)$. The intensity profile for the propagating modes of the waveguide for the chosen set of parameter is shown in the lower panel of Fig. 1(b) where the green line represents the fundamental mode and the black line represents the higher-order mode.

With the onset of gain-loss, the supported modes (say, $\psi_{\mathrm{FM}}$ and $\left.\psi_{\mathrm{HOM}}\right)$ are mutually coupled. Here, to encounter an EP, we exploit the concept of avoided resonance crossings (ARC) phenomena $[8,9,14,17]$ between the corresponding propagation constants (say, $\beta_{\mathrm{FM}}$ and $\beta_{\mathrm{HOM}}$ ) with crossing and anticrossing of their real and imaginary parts, i.e., $\operatorname{Re}(\beta)$ and $\operatorname{Im}(\beta)$. Now varying the parameter $\gamma$ within a chosen range $[0,0.015]$, we study such ARC-interactions phenomena between $\beta_{\mathrm{FM}}$ and $\beta_{\mathrm{HOM}}$ for different $\tau$-values. Judiciously examining the several cases, we set a specific $\tau=3.179$, for which, $\beta_{\mathrm{FM}}$ and $\beta_{\mathrm{HOM}}$ coalesce in the $\beta$-plane near $\gamma \approx 0.008$ as can be seen in Fig. 1(c), that refers the presence of an EP in the $(\gamma, \tau)$-plane, around which we can observe two topologically different ARC phenomena. Thus, numerically we identify an EP at $\sim\left(\gamma_{\mathrm{EP}}=0.008, \tau_{\mathrm{EP}}=3.179\right)$.

Now, to encircle the identified EP, we consider a closed parameter space in the $(\gamma, \tau)$-plane following the equations

$$
\gamma(\phi)=\gamma_{0} \sin \left(\frac{\phi}{2}\right) ; \quad \tau(\phi)=\tau_{\mathrm{EP}}+a \sin (\phi) .
$$

Here $\gamma_{0}$ and $a$ are two characteristic parameters, and $\phi(0 \leqslant$ $\phi \leqslant 2 \pi)$ is a tunable angle variable that govern the variation of $\gamma$ and $\tau$ around the EP. Here, to ensure the presence of EP inside the parametric loop, we must consider $\gamma_{0}>\gamma_{\text {EP }}$. Here, the variation of $\phi$ from 0 to $2 \pi$ gives the clockwise progression of $\gamma$ and $\tau$. On the other hand, the variation of $\phi$ from $2 \pi$ to 0 gives the anticlockwise progression of $\gamma$ and $\tau$. Unlike the conventional circular loops, this specific shape of the parametric-loop facilitates the device to achieve passive modes at the input and output interface $[9,15,17]$. Now, to consider the dynamical encirclement and to realize the actual beam propagation through the waveguide around an EP, we have to map this parameter space [given by Eq. (5)] along the $z$-direction through the complete length $(L)$ of the waveguide. For this mapping, we choose $\phi=2 \pi z / L$ to consider $\phi=$ 0 at $z=0$ and $\phi=2$ at $z=L$. Thus the length-dependent variation of $\gamma$ and $\tau$ can be written as

$$
\gamma(z)=\gamma_{0} \sin \left[\frac{\pi z}{L}\right] ; \quad \tau(z)=\tau_{\mathrm{EP}}+a \sin \left[\frac{2 \pi z}{L}\right] .
$$

Such a length-dependent parameter space is shown in Fig. 1(d) with $\gamma_{0}=0.009\left(>\gamma_{\mathrm{EP}}\right)$ and $a=0.5$. The shape of the corresponding closed-loop in the $(\gamma, \tau)$-plane [following Eq. (5)] is shown in the inset. Thus, a complete encirclement around the EP following Eq. (5) is equivalent to one complete pass of light along the length of the waveguide; where the clockwise encirclement is equivalent to the propagation from $z=0$ to $z=L$, i.e., the forward propagation, and the anticlockwise encirclement is equivalent to the propagation from $z=L$ to $z=0$, i.e., the backward propagation. Now, such propagations of the modes through the waveguide should follow the time-dependent Schrödinger equation (TDSE), where time is the quantum-mechanical counterpart of the $z$-axis. To study the modal propagation, we use scalar beam propagation to solve the equation $2 i \omega \partial_{z} \psi(x, z)=-\left[\partial_{x}^{2}+\right.$ $\left.\Delta n^{2}(x, z) \omega^{2}\right] \psi(x, z)\left[\Delta n^{2}(x, y) \equiv n^{2}(x, z)-n_{l}^{2}\right]$, taking into account the paraxial approximation and the adiabatic variation of $\operatorname{Im}(n)$ in the $z$-axis.

The light dynamics in the designed waveguide following the dynamical EP encirclement scheme [described in Fig. 1(d)] are shown in Figs. 1(e) and 1(f). Light is launched at $z=0$ for the implementation of a clockwise encirclement scheme, which yields the conversion of both $\psi_{\mathrm{FM}}$ and $\psi_{\mathrm{HOM}}$ to $\psi_{\mathrm{HOM}}$ at $z=L$ as shown in plots (e.1) and (e.2) of Fig. 1(e). Here, we have one nonadiabatic transition (NAT) corresponding to $\psi_{\mathrm{HOM}}$. Now, to implement an anticlockwise encirclement scheme, we launch the light at $z=L$, which yields the conversion of both $\psi_{\mathrm{FM}}$ and $\psi_{\mathrm{HOM}}$ to $\psi_{\mathrm{FM}}$, as shown in Fig. 1(f). Here $\psi_{\mathrm{HOM}}$ is converted adiabatically to $\psi_{\mathrm{FM}}$ and $\psi_{\text {FM }}$ follows a NAT. Thus, we observe a breakdown in adiabaticity in modal evolutions due to the dynamical variation of the control parameters $(\gamma$ and $\tau$ ) around the EP $[15,17,31,32]$ that results in a chirality-driven asymmetric mode conversion phenomenon, where regardless of the choice of inputs, light is converted in a specific mode depending the direction of propagation.

\section{EFFECTS OF NONLINEARITY ON THE BEAM DYNAMICS}

Now, we introduce local Kerr nonlinearity as an additional parameter along with optical gain-loss in the waveguide to control the interactions between the two supported modes. The Kerr nonlinearity has the form $\Delta n_{\mathrm{NL}}(x, y)=\sigma n_{2} I$, where $n_{2}$ is the nonlinear coefficient, $I$ is the signal intensity, and $\sigma=+1$ for focusing nonlinearity and $\sigma=-1$ for defocusing nonlinearity. In a prototype of the proposed waveguide, the local nonlinearity distribution can be achieved through the intensity $(I)$ of the injected light-signal, and varying the signal power, we can change the nonlinearity amounts. In a generalized form, the nonlinearity amounts can also be quantified with respect to $\Delta n\left(=n_{h}-n_{l}\right)$ through the percentage of nonlinearities as $\left(\Delta n_{\mathrm{NL}} / \Delta n\right) \times 100 \%$. During the numerical investigation of the dynamics of the eigenmodes, we choose the appropriate value of the nonlinear coefficient $\left(n_{2}\right)$ of silicabased materials and launch the light signal with an initial power of 80 watts and vary the signal power in between 80 to 120 watts to achieve the nonlinearity levels up to $6 \%$.

While introducing nonlinearity below $1 \%$, we do not observe any effect of nonlinearity on the overall phenomenon 

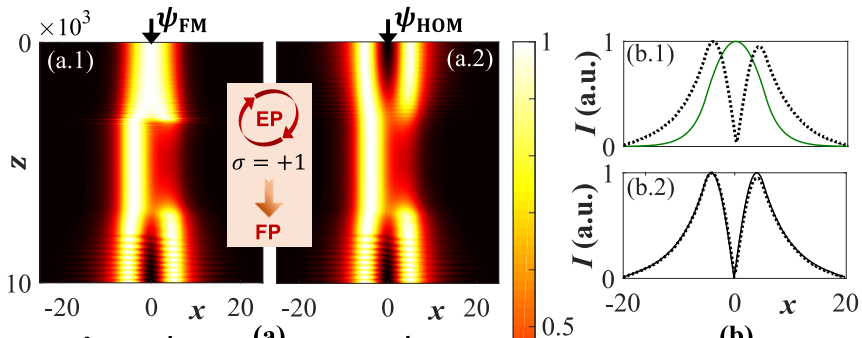

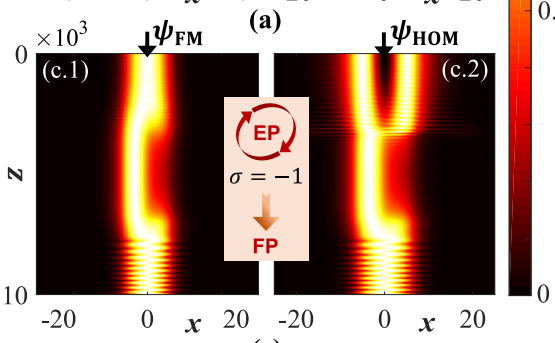

(c)

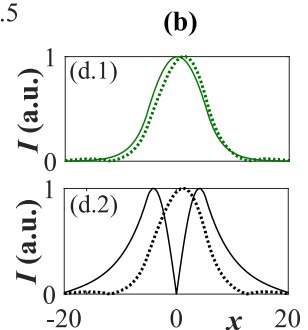

(d)
FIG. 2. Beam propagation results in presence of $3 \%$ nonlinearity. (a) The conversions $\psi_{\mathrm{FM}} \rightarrow \psi_{\mathrm{HOM}}$ (plot a.1) and $\psi_{\mathrm{HOM}} \rightarrow \psi_{\mathrm{HOM}}$ (plot a.2) in the presence of focusing nonlinearity (FN) while encircling the EP in the clockwise direction. (b) Plots (b.1) and (b.2) show the normalized field intensities at $z=0$ (solid curves indicating the inputs) and $z=L$ (dotted curves indicating the outputs) corresponding to the beam propagation shown in plots (a.1) and (a.2), respectively. (c) Beam propagation results showing the conversions $\psi_{\mathrm{FM}} \rightarrow \psi_{\mathrm{FM}}$ (plot c.1) and $\psi_{\mathrm{HOM}} \rightarrow \psi_{\mathrm{FM}}$ (plot c.2) in the presence of defocusing nonlinearity (DFN) while encircling the EP in the clockwise direction. (d) Plots (d.1) and (d.2) show the normalized field intensities at $z=0$ (solid curves indicating the inputs) and $z=L$ (dotted curves indicating the outputs) corresponding to the beam propagation shown in plots (c.1) and (c.2), respectively.

of asymmetric mode conversion, as described in the preceding section. Here the EP-aided nonadiabatic corrections essentially control the overall light dynamics through the waveguide, where the conversion of the eigenmodes depends on the direction of propagation of light. A completely different scenario is observed when we increase nonlinearity above this threshold value. Now implementing a clockwise encirclement scheme where we consider the propagation of light from $z=0$ to $z=L$; initially, we choose $\sigma=1$ for focusing nonlinearity. After reaching nonlinearity of 3\%, both the modes collapse to $\psi_{\mathrm{HOM}}$, as shown in Figs. 2(a.1) and 2(a.2), respectively. In the plots (b.1) and (b.2) of Fig. 2(b), we show the normalized field intensities at two different ports of the waveguide corresponding to the beam propagation shown in plots (a.1) and (a.2), respectively of Fig. 2(a). Here the solid and dotted curves of respective colors (green for $\psi_{\mathrm{FM}}$ and black for $\psi_{\mathrm{HOM}}$ ) represent the input and output intensities. Next, by choosing $\sigma=-1$ for defocusing nonlinearity, we study the modal dynamics with the onset of the same amount of nonlinearity as before and observe the conversion of both the modes to $\psi_{\mathrm{FM}}$, which can be seen in Figs. 2(c.1) and 2(c.2). The corresponding normalized output intensities to the beam propagation shown in the plots (c.1) and (c.2) of Fig. 2(c) are shown by the dotted green and black lines in Figs. 2(d.1) and 2(d.2).

Now, to change the direction of propagation of light, we implement an anticlockwise encirclement scheme and study

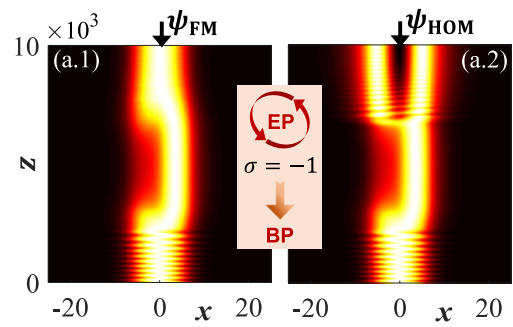

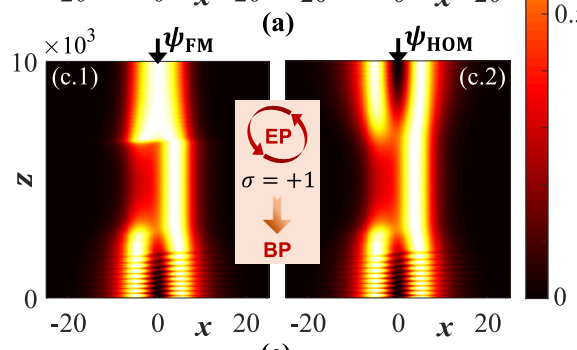

(c)

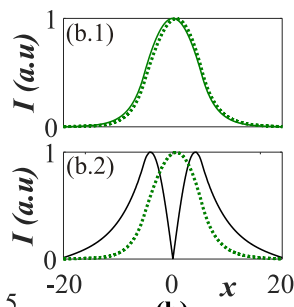

(b)

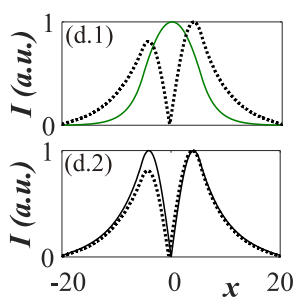

(d)
FIG. 3. Beam propagation results in presence of $5 \%$ nonlinearity. (a) The conversions $\psi_{\mathrm{FM}} \rightarrow \psi_{\mathrm{FM}}$ (plot a.1) and $\psi_{\mathrm{HOM}} \rightarrow \psi_{\mathrm{FM}}$ (plot a.2) in the presence of DFN while encircling the EP in the anticlockwise direction. (b) Plots (b.1) and (b.2) show the normalized field intensities at $z=0$ (solid curves indicating the inputs) and $z=L$ (dotted curves indicating the outputs) corresponding to the beam propagation shown in plots (a.1) and (a.2), respectively. (c) Beam propagation results showing the conversions $\psi_{\mathrm{FM}} \rightarrow \psi_{\mathrm{HOM}}$ (plot c. 1) and $\psi_{\mathrm{HOM}} \rightarrow \psi_{\mathrm{HOM}}$ (plot c.2) in the presence of FN while encircling the EP in the anticlockwise direction. (d) Plots (d.1) and (d.2) show the normalized field intensities at $z=0$ (solid curves indicating the inputs) and $z=L$ (dotted curves indicating the outputs) corresponding to the beam propagation shown in plots (c.1) and (c.2), respectively.

the modal dynamics. Considering a defocusing-type nonlinearity $(\sigma=-1)$, we increase the amount of nonlinearity up to $5 \%$, where it is observed that both the eigenmodes collapse to $\psi_{\mathrm{FM}}$, as shown in the plots (a.1) and (a.2) of Fig. 3(a), respectively. The plots (b.1) and (b.2) of Fig. 3 show the normalized input (solid green and black lines for the FM and HOM, respectively) and output intensities (dotted lines of respective colors) for the propagations shown in Figs. 3(a.1) and 3(a.2). Again, the onset of the same amount of focusing nonlinearity $(\sigma=1)$ yields the conversion of both the eigenmodes to $\psi_{\mathrm{HOM}}$, as shown in plots (c.1) and (c.2) of Fig. 3(c). In a similar way, the corresponding input-output intensities are shown in plots (d.1) and (d.2) of Fig. 3.

Thus, with an unconventional dynamical EP-encirclement scheme in the presence of local Kerr nonlinearity (above a specific threshold) in the spatial distribution of refractive index of the waveguide a nonchiral, unidirectional asymmetric mode conversion scheme (as illustrated in Figs. 2 and 3) is proposed that is entirely different from the EP-aided chiralitydriven asymmetric-mode-conversion scheme [as shown in Figs. 1(e) and 1(f)], in the absence of nonlinearity. The proposed waveguide exhibits a chirality-driven asymmetric mode conversion scheme in the absence of nonlinearity, where, at the output, the waveguide delivers $\psi_{\mathrm{HOM}}$ during the propagation in the forward direction, whereas $\psi_{\mathrm{FM}}$ during the backward propagation, irrespective of the choice of inputs. Thus, the dominating output depends on the device chirality, i.e., the 

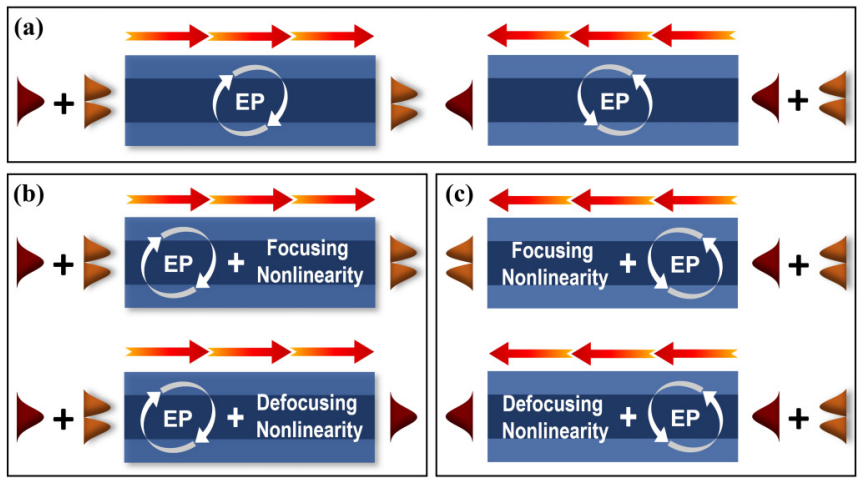

FIG. 4. Schematic representation of the overall scheme explored in this work. (a) The chirality-driven asymmetric mode conversion scheme in the presence of nonlinearity below the threshold level (or in the absence of nonlinearity). Nonchiral unidirectional asymmetric mode conversion schemes (b) for 3\% nonlinearity (high transmission in the forward direction) and (c) for 5\% nonlinearity (high transmission in the backward direction). The group of red arrows indicate the direction of light propagation.

direction of light propagation. For our specific configuration, we find a threshold nonlinearity amount at $1 \%$, i.e., below $1 \%$ nonlinearity level, the waveguide holds the standard EP-aided chiral behavior. However, once the nonlinearity level exceeds its threshold amount, the waveguide enables unidirectional asymmetric mode conversion. The presence of nonlinearity above its threshold amount induce a notable difference in transmission levels during the propagation of light in two different directions [24]. Here, we observe that in the presence of $3 \%$ local nonlinearity, the waveguide offers high transmission in the forward direction and low transmission in the backward direction. During the forward propagation of light, the same amount of focusing-type nonlinearity yields $\psi_{\mathrm{HOM}}$, whereas defocusing-type nonlinearity yields $\psi_{\mathrm{FM}}$, at the output. Thus, without changing the direction of light propagation, we get a different dominating mode at the same output port that yields a nonchiral (i.e., beyond the chiral aspect of the device) asymmetric mode conversion phenomenon. Now, if we consider backward propagation of light under this operating condition, then we get similar outputs for focusing and defocusing nonlinearity, however, for $3 \%$ nonlinearity, the transmission becomes low in the backward direction. We found an average of 2-dB difference between forward and backward transmissions. Thus, we further optimize the nonlinearity amount by varying the signal intensity to check the device applicability in the backward direction and find that when we increase the nonlinearity to $5 \%$, the proposed waveguide offers high transmission in the backward direction and low transmission in the forward direction. Under this operating condition, during the backward propagation of light, the same amount of focusing-type nonlinearity yields $\psi_{\mathrm{HOM}}$, whereas defocusing-type nonlinearity yields $\psi_{\mathrm{FM}}$, at the output. The overall observation is shown schematically in Fig. 4, where in Fig. 4(a), the chirality driven asymmetric mode conversion scheme in the absence of nonlinearity (or the presence of nonlinearity amount below the threshold level) is illustrated, and in Figs. 4(b) and 4(c), two nonchiral unidirectional asymmetric mode conversions are demonstrated for two different nonlinearity amounts for which the waveguide shows high transmission in two different directions.

In the proposed scheme, the nonchiral asymmetric mode conversion is independent of the direction of light propagation (or direction of EP encirclement), whereas it mainly depends on the type of nonlinearity. For a specific nonlinearity type, there is the breakdown in the chirality of the device because here the waveguide delivers $\psi_{\mathrm{HOM}}$ in the presence of focusing nonlinearity, whereas $\psi_{\mathrm{FM}}$ in the presence of defocusing nonlinearity, irrespective of the direction of light propagation (however, to get high transmission we have to choose two different nonlinearity amounts, while considering forward and backward propagation), and also the choice of inputs.

\section{ANALYTICAL APPROACH TOWARD THE NONADIABATIC CORRECTIONS IN THE BEAM DYNAMICS}

The analytical treatment behind such nonadiabatic state evolution of one of the two eigenstates is presented here [17,31]. We assume the $2 \times 2$ Hamiltonian $\mathcal{H}(t)$, given in Eq. (1), depends on two generic time-dependent potential parameters $\kappa_{1}(t)$ and $\kappa_{2}(t)$ that includes the simultaneous effect of gain-loss and nonlinearity. However, the effect of nonlinearity comes into the picture only when nonlinearity amount exceeds its threshold level (1\% for the proposed waveguide configuration). Now, instead of $\beta_{ \pm}$, here we consider the physical eigenvalues $\beta_{\mathrm{FM}}^{a d}\left(\kappa_{1}, \kappa_{2}\right)$ and $\beta_{\mathrm{HOM}}^{a d}\left(\kappa_{1}, \kappa_{2}\right)$ with corresponding eigenvectors $\psi_{\mathrm{FM}}^{a d}\left(\kappa_{1}, \kappa_{2}\right)$ and $\psi_{\mathrm{HOM}}^{a d}\left(\kappa_{1}, \kappa_{2}\right)$ to represent the proposed waveguide. The time dependence of the potential parameters $\kappa_{1}(t)$ and $\kappa_{2}(t)$ governs the dynamical nonadiabatic corrections in the solutions of the TDSE associated with the Hamiltonian $\mathcal{H}(t)$. Such nonadiabatic correction terms due to dynamical encirclement around the EP can be written as

$$
\begin{aligned}
& \mathcal{M}_{\mathrm{F} \rightarrow \mathrm{H}}^{\mathrm{NA}}=C_{\mathrm{F} \rightarrow \mathrm{H}} \exp \left\{-i \oint_{0}^{T} \Delta \beta_{\mathrm{F}, \mathrm{H}}^{a d}\left[\kappa_{1}, \kappa_{2}\right] d t\right\}, \\
& \mathcal{M}_{\mathrm{H} \rightarrow \mathrm{F}}^{\mathrm{NA}}=C_{\mathrm{H} \rightarrow \mathrm{F}} \exp \left\{+i \oint_{0}^{T} \Delta \beta_{\mathrm{F}, \mathrm{H}}^{a d}\left[\kappa_{1}, \kappa_{2}\right] d t\right\},
\end{aligned}
$$

with the pre-exponent terms

$$
\begin{aligned}
& C_{\mathrm{F} \rightarrow \mathrm{H}}=\left\langle\psi_{\mathrm{FM}}^{a d}\left(\kappa_{1}, \kappa_{2}\right)\left|\sum_{j=1}^{2} \dot{\kappa_{j}} \frac{\partial}{\partial \kappa_{j}}\right| \psi_{\mathrm{HOM}}^{a d}\left(\kappa_{1}, \kappa_{2}\right)\right\rangle, \\
& C_{\mathrm{H} \rightarrow \mathrm{F}}=\left\langle\psi_{\mathrm{HOM}}^{a d}\left(\kappa_{1}, \kappa_{2}\right)\left|\sum_{j=1}^{2} \dot{\kappa_{j}} \frac{\partial}{\partial \kappa_{j}}\right| \psi_{\mathrm{FM}}^{a d}\left(\kappa_{1}, \kappa_{2}\right)\right\rangle .
\end{aligned}
$$

The factor $\Delta \beta_{\mathrm{F}, \mathrm{H}}^{a d}$ [in the exponent terms of Eq. (7)] can be expressed as

$$
\begin{aligned}
\Delta \beta_{\mathrm{F}, \mathrm{H}}^{a d}\left(\kappa_{1}, \kappa_{2}\right) & =\beta_{\mathrm{FM}}^{a d}\left(\kappa_{1}, \kappa_{2}\right)-\beta_{\mathrm{HOM}}^{a d}\left(\kappa_{1}, \kappa_{2}\right) \\
& \equiv \operatorname{Re}\left[\Delta \beta_{\mathrm{F}, \mathrm{H}}^{a d}\left(\kappa_{1}, \kappa_{2}\right)\right]-i \Delta \gamma_{\mathrm{F}, \mathrm{H}}^{a d}\left(\kappa_{1}, \kappa_{2}\right) .
\end{aligned}
$$

In Eq. (7), $\mathcal{M}_{\mathrm{F} \rightarrow \mathrm{H}}^{\mathrm{NA}}$ and $\mathcal{M}_{\mathrm{H} \rightarrow \mathrm{F}}^{\mathrm{NA}}$ represent the nonadiabatic correction factor for the conversion of $\left|\psi_{\mathrm{FM}}^{a d}\right\rangle$ to $\left|\psi_{\mathrm{HOM}}^{a d}\right\rangle$ and vice versa. $T$ is the duration of EP encirclement and $\left|\Delta \gamma_{\mathrm{F}, \mathrm{H}}^{a d}\right|$ is the relative gain between the supported modes. Since the 
pre-exponent terms given by Eq. (8) contain the time derivative of the two potential parameters $\dot{\kappa}_{j}(j=1,2)$, the divergence in $T$ due to the exponential terms of $\mathcal{M}_{\mathrm{F} \rightarrow \mathrm{H}}^{\mathrm{NA}}$ and $\mathcal{M}_{\mathrm{H} \rightarrow \mathrm{F}}^{\mathrm{NA}}$ surpasses the decay of $T^{-1}$ incorporated in $C_{\mathrm{F} \rightarrow \mathrm{H}}$ and $C_{\mathrm{H} \rightarrow \mathrm{F}}$, respectively. Thus, for the situations, $\Delta \gamma_{\mathrm{F}, \mathrm{H}}^{a d}>0$ and $T \rightarrow \infty$, the correction terms $\mathcal{M}_{\mathrm{F} \rightarrow \mathrm{H}}^{\mathrm{NA}} \rightarrow 0$ and $\mathcal{M}_{\mathrm{H} \rightarrow \mathrm{F}}^{\mathrm{NA}} \rightarrow \infty$. Here, the situation $\mathcal{M}_{\mathrm{F} \rightarrow \mathrm{H}}^{\mathrm{NA}} \rightarrow 0$ yields the adiabatic evolution of $\left|\psi_{\mathrm{FM}}^{a d}\right\rangle$, and the situation $\mathcal{M}_{\mathrm{H} \rightarrow \mathrm{F}}^{\mathrm{NA}} \rightarrow \infty$ yields the nonadiabatic evolution of $\left|\psi_{\mathrm{HOM}}^{a d}\right\rangle$. Therefore, under a slow parametric variation encircling an EP, out of the two considered modes, only one mode with an overall lower decay rate undergoes with the adiabatic evolution whereas the other one evolves nonadiabatically.

Now, we consider our proposed waveguide configuration in the absence of nonlinearity (or in the presence of nonlinearity below the threshold amount). Here, while considering the clockwise dynamical encirclement scheme, we obtain $\mathcal{M}_{\mathrm{F} \rightarrow \mathrm{H}}^{\mathrm{NA}} \rightarrow 0$ and $\mathcal{M}_{\mathrm{H} \rightarrow \mathrm{F}}^{\mathrm{NA}} \rightarrow \infty$, and accordingly, the mode $\psi_{\mathrm{FM}}$ evolves adiabatically and converted to $\psi_{\mathrm{HOM}}$, whereas, $\psi_{\mathrm{HOM}}$ evolves nonadiabatically and remains in $\psi_{\mathrm{HOM}}$, as can be seen in Fig. 1(e). In a similar way, for the anticlockwise dynamical encirclement scheme, we obtain $\mathcal{M}_{\mathrm{F} \rightarrow \mathrm{H}}^{\mathrm{NA}} \rightarrow \infty$ and $\mathcal{M}_{\mathrm{H} \rightarrow \mathrm{F}}^{\mathrm{NA}} \rightarrow 0$ that yields nonadiabatic evolution of $\psi_{\mathrm{FM}}(\rightarrow$ $\left.\psi_{\mathrm{FM}}\right)$ and adiabatic conversion of $\psi_{\mathrm{HOM}}$ to $\psi_{\mathrm{FM}}$, as can be seen in Fig. 1(f).

Now, in the presence of nonlinearity above the threshold level, the desired outputs for the nonchiral unidirectional asymmetric mode conversions shown in Figs. 2 and 3 can also be predicted analytically. The application of the different types of nonlinearity in the optical waveguide modifies the refractive index profile locally, which in turn changes the $\beta$-values of the quasiguided modes, and accordingly, the sign of relative-gain factor $\Delta \gamma_{\mathrm{F}, \mathrm{H}}^{a d}$ in Eq. (9) are also modified. Here, for both the optimized nonlinearity levels, when we consider focusing-type nonlinearity, then we find that $\Delta \gamma_{\mathrm{F}, \mathrm{H}}^{a d}>0$, irrespective of the direction of light propagation. This criterion gives the conversions of both $\psi_{\mathrm{FM}}$ and $\psi_{\mathrm{HOM}}$ to $\psi_{\mathrm{HOM}}$, as can be seen in Figs. 2(a) and 2(b) and
Figs. 3(c) and 3(d), respectively. Here, $\psi_{\mathrm{FM}}$ evolves adiabatically and $\psi_{\text {HOM }}$ behaves nonadiabatically. However, while we consider defocusing-type nonlinearity, for both the optimized nonlinearity amounts, we obtain $\Delta \gamma_{\mathrm{F}, \mathrm{H}}^{a d}<0$, irrespective of the direction of light propagation. Accordingly, $\psi_{\mathrm{FM}}$ behaves nonadiabatically and remains in $\psi_{\mathrm{FM}}$, whereas $\psi_{\mathrm{HOM}}$ evolves adiabatically and is converted to $\psi_{\mathrm{FM}}$ [as shown in Figs. 2(c) and 2(d) and 3(a) and 3(b), respectively).

\section{CONCLUSION}

In summary, we report a gain-loss assisted dual-mode optical waveguide hosting dynamical EP encirclement scheme and study the effect of Kerr nonlinearity on the associated light dynamics around the EP. The waveguide exhibits a chirality-driven asymmetric-mode-conversion, in the absence of nonlinearity. Now, when we introduce local Kerr nonlinearity above a particular threshold, it locally modifies the spatial index distribution and then the waveguide enables a unique unidirectional asymmetric-mode-conversion scheme. Here, beyond the device chirality, light is converted to a specific dominating mode irrespective of the direction of propagation, where the same amount of focusing $(\sigma=1)$ and defocusing $(\sigma=-1)$ nonlinearities yields a different dominating output in the same direction. The proposed scheme will undoubtedly facilitate the fabrication possibilities of optical mode converters, switches and isolators in chip-based devices for next-generation photonic circuits.

\section{ACKNOWLEDGMENTS}

J.D. acknowledges financial support from University Grants Commission (UGC), India. A.L. and S.G. acknowledge financial support from the Science and Engineering Research Board (SERB), India (Early Career Research Scheme, Grant No. ECR/2017/000491). S.D. acknowledges support from the Ministry of Human Resource Development (MHRD), India.
[1] N. Moiseyev, Non-Hermitian Quantum Mechanics (Cambridge University Press, New York, 2011).

[2] M. A. Miri and A. Alù, Science 363, 6422 (2019).

[3] W. D. Heiss, Phys. Rev. E 61, 929 (2000).

[4] W. D. Heiss and A. L. Sannino, J. Phys. A: Math. Gen. 23, 1167 (1990).

[5] T. Kato, Perturbation Theory of Linear Operators (Springer, Berlin, 1995).

[6] W. D. Heiss, J. Phys. A: Math. Theor. 45, 444016 (2012).

[7] C. Dembowski, B. Dietz, H.-D. Gräf, H. L. Harney, A. Heine, W. D. Heiss, and A. Richter, Phys. Rev. E 69, 056216 (2004).

[8] A. Laha, A. Biswas, and S. Ghosh, J. Opt. Soc. Am. B 34, 2050 (2017).

[9] A. Laha, A. Biswas, and S. Ghosh, J. Opt. (IOP) 21, 025201 (2019).

[10] S. Bhattacherjee, A. Laha, and S. Ghosh, Phys. Scr. 94, 085202 (2019).
[11] S. Bhattacherjee, A. Laha, and S. Ghosh, Phys. Scr. 94, 105509 (2019).

[12] H. Menke, M. Klett, H. Cartarius, J. Main, and G. Wunner, Phys. Rev. A 93, 013401 (2016).

[13] H. Cartarius, J. Main, and G. Wunner, Phys. Rev. Lett. 99, 173003 (2007).

[14] A. Laha and S. Ghosh, J. Opt. Soc. Am. B 34, 238 (2017).

[15] S. Ghosh and Y. D. Chong, Sci. Rep. 6, 19837 (2016).

[16] J. Doppler, A. A. Mailybaev, J. Böhm, U. Kuhl, A. Girschik, F. Libisch, T. J. Milburn, P. Rabl, N. Moiseyev, and S. Rotter, Nature 537, 76 (2016).

[17] A. Laha, A. Biswas, and S. Ghosh, Phys. Rev. Applied 10, 054008 (2018).

[18] H. K. Gandhi, A. Laha, S. Dey, and S. Ghosh, Opt. Lett. 45, 1439 (2020).

[19] H. Xu, D. Mason, L. Jiang, and J. G. E. Harris, Nature 537, 80 (2016). 
[20] Z. J. Wong, Y.-L. Xu, J. Kim, K. O’Brien, Y. Wang, L. Feng, and X. Zhang, Nat. Photon. 10, 796 (2016).

[21] X. Yin and X. Zhang, Nat. Mater. 12, 175 (2013).

[22] J. Wiersig, Phys. Rev. A 93, 033809 (2016).

[23] R. Thomas, H. Li, F. M. Ellis, and T. Kottos, Phys. Rev. A 94, 043829 (2016).

[24] Y. Choi, C. Hahn, J. W. Yoon, S. H. Song, and P. Berini, Nat. Commun. 8, 14154 (2017).

[25] A. Laha, S. Dey, H. K. Gandhi, A. Biswas, and S. Ghosh, ACS Photonics 7, 967 (2020).

[26] T. Goldzak, A. A. Mailybaev, and N. Moiseyev, Phys. Rev. Lett. 120, 013901 (2018).

[27] D. A. Bykov and L. L. Doskolovich, Phys. Rev. A 97, 013846 (2018).

[28] W. D. Heiss and G. Wunner, J. Phys. A: Math. Theor. 48, 345203 (2015).
[29] B. Midya and V. V. Konotop, Phys. Rev. Lett. 119, 033905 (2017).

[30] J. Schnabel, H. Cartarius, J. Main, G. Wunner, and W. D. Heiss, Phys. Rev. A 95, 053868 (2017).

[31] I. Gilary, A. A. Mailybaev, and N. Moiseyev, Phys. Rev. A 88, 010102(R) (2013).

[32] R. Uzdin, A. Mailybaev, and N. Moiseyev, J. Phys. A: Math. Theor. 44, 435302 (2011).

[33] X.-L. Zhang and C. T. Chan, Commun. Phys. 2, 63 (2019).

[34] X.-L. Zhang, S. Wang, B. Hou, and C. T. Chan, Phys. Rev. X 8, 021066 (2018).

[35] A. Pick, Z. Lin, W. Jin, and A. W. Rodriguez, Phys. Rev. B 96, 224303 (2017).

[36] S. Phang, A. Vukovic, S. C. Creagh, T. M. Benson, P. D. Sewell, and G. Gradoni, Opt. Express 23, 11493 (2015). 